\title{
Se è sindacale spetta al sindaco o al sindacato?
}

\section{Paolo D'Achille}

PUBBLICATO: 25 FEBBRAIO 2020

\section{Quesito:}

Ci sono pervenute varie domande che chiedono se è corretta l'espressione ordinanza sindacale, che si trova spesso negli atti delle amministrazioni comunali per indicare una disposizione emanata dal sindaco, o se non possa generare confusione, visto che l'aggettivo sindacale è normalmente riferito al sindacato (rappresentante sindacale, contributo sindacale, ecc.).

\section{Se è sindacale spetta al sindaco o al sindacato?}

I dizionari contemporanei (Sabatini-Coletti, GRADIT, Zingarelli 2020, ecc.) distinguono due omonimi. Esiste un primo aggettivo sindacale, riferito al sindaco di un Comune, cioè al capo di un'amministrazione comunale, eletto direttamente dai cittadini (a cui si riferisce l'ordinanza sindacale citata dai nostri lettori), oppure, meno spesso, al sindaco di una società privata, che ha il compito di controllare l'amministrazione, vigilare sullosservanza della legge, ecc. (in genere i sindaci sono più di uno, tanto che si parla di collegio sindacale). Il termine sindaco, infatti, in italiano antico significava (cfr. DELI) non solo 'rappresentante del comune', ma anche 'chi agisce in nome e nell'interesse d'un altro' e 'chi rivede i conti'.

C'è poi un altro aggettivo sindacale, che si riferisce al sindacato, cioè a un'organizzazione che associa i membri di una categoria di lavoratori, per rappresentarne e difenderne gli interessi economici e professionali: si parla infatti di organizzazioni sindacali, rappresentanze e rappresentanti sindacali, contributo sindacale, ecc.

Questo secondo sindacale, riferito al sindacato, è d'uso comune molto più del primo, quello riferito al sindaco (il che spiega perché l'espressione ordinanza sindacale sia percepita come "strana" dai nostri lettori), e, diversamente da questo, ha prodotto vari derivati (antisindacale, sindacalese 'il linguaggio tipico dei sindacati', sindacalismo, sindacalista, sindacalizzare). Non immotivatamente, dunque, il Sabatini-Coletti e il GRADIT (diversamente dallo Zingarelli 2020) lo registrano per primo, come ${ }^{1}$ sindacale. L'altro sindacale è però più antico: lo Zingarelli 2020 lo data al 1676 , anticipando notevolmente il GRADIT e il DELI (I872), mentre tutti i dizionari concordano nel datare il sindacale riferito al sindacato al I905, sulla base del volume di Luigi Campolonghi, Azione sindacale (Roma, Mongini) citato nel DELI.

Il sindacale più antico si deve considerare un derivato, col suffisso-ale, di sindaco, termine (come si è detto) attestato già in italiano antico, a partire dal Duecento (allora il plurale era sindachi, non sindaci), che deriva dal latino tardo syndìcu(m) (a sua volta dal greco sýndikos 'difensore pubblico', derivato da díke 'giustizia', col prefisso sýn- 'insieme') e presenta (come rileva l'Etimologico) la stessa evoluzione fonetica della $\iota$ postonica in $a$ che si ha in cronaca (derivato dal latino chronica).

Da sindaco deriva anche sindacato nel senso (documentato già nel Duecento) di 'rendiconto dovuto da alcuni magistrati o amministratori ai loro superiori' e che poi, più in generale, ha assunto il significato di 'controllo dell'attività di un subordinato oppure di un ente, un organismo'. Come sindacale, dunque, anche sindacato ha un omonimo piu antico, probabilmente derivato dal verbo sindacare, 
documentato dalla fine del Duecento nel senso di 'esaminare attentamente, controllare l'operato di persone, enti, amministrazioni' e sim.' e poi in quello di 'sottoporre a controlli o critiche', tuttora ben diffuso (in genere in senso non positivo: perché hai sempre da sindacare su quello che dico o che faccio?), da cui più tardi sono derivati gli aggettivi sindacabile e insindacabile, che significano, rispettivamente, 'che può o non può essere messo in discussione'.

Lasciamo da parte tanto l'uso aggettivale di sindacato participio passato di sindacare quanto l'esistenza di un secondo sindacare tratto da sindacato nel senso di 'iscrivere a un'associazione sindacale' (lo Zingarelli 2020 lo marca come disusato in questo senso, ma ne registra un senso specifico nel settore dell'economia) per segnalare invece che c'è un altro sindacato ancora, d'uso molto più circoscritto, anch'esso derivato da sindaco, che significa 'ufficio di sindaco' o 'durata della carica di sindaco'. Lo Zingarelli 2020 lo data al I887, ma è documentato, come si ricava da Google Libri, già nel vol. IV della Legislazione toscana a cura di Lorenzo Cantini (Firenze, Stamperia Albizziana, I802).

Invece sindacato nel senso sopra ricordato di 'organizzazione di gruppi di lavoratori' (datato igoo nel GRADIT, che registra anche altre accezioni più specifiche, una delle quali attestata già nel I895, citate anche nello Zingarelli 2020, su cui ora non ci soffermiamo) e sindacale nel senso di 'relativo al sindacato' sono calcati sui nuovi significati assunti, nel corso del sec. XIX, dai corrispondenti termini francesi syndicat e syndical, che hanno, alla lontana, la stessa derivazione dei nostri sindacato e sindacale (secondo il DELI syndical, attestato in francese dal ı7or, "aveva all'inizio [...] il sign[ificato] di 'fatto alla comunità, in opposizione alle azioni private (il che spiega i sign[ificati] moderni di questa famiglia di voci)"), ma non presentano l'evoluzione della $\iota$ postonica in $a$, estranea al francese (che infatti in corrispondenza del nostro cronaca ha chronique). I calchi italiani di queste parole francesi sono stati influenzati dalle voci italiane già esistenti e così le forme sindacato e sindacale hanno prevalso sulle varianti sindicato e sindicale (entrambe ben documentate nel corso dell'Ottocento), determinando questa doppia (tripla nel caso di sindacato) omonimia.

Un'omonimia non felicissima, certo, trattandosi di termini che rientrano tutti, in prima istanza, nel lessico burocratico-amministrativo, ma che la lingua riesce a tollerare affidandosi ai contesti e alle collocazioni, che permettono di capire di volta in volta se sindacale si riferisce a un sindacato (come è certo assai più frequente) o a un sindaco (o a una sindaca).

In conclusione, ordinanza sindacale è espressione senz'altro legittima e corretta, ma forse si potrebbero invitare le amministrazioni comunali a non abusarne, almeno nei manifesti e negli avvisi pubblici (tanto più che anche ordinanza è oggi termine d'uso solo settoriale, di ambito giuridico o militare): disposizione del sindaco o provvedimento del sindaco sarebbero per i cittadini espressioni senz'altro più semplici e più chiare.

\section{Cita come:}

Paolo D'Achille, Se è sindacale spetta alsindaco o alsindacato?, "Italiano digitale", 2019, XII, 2020/1 (gennaio-marzo)

DOI: $10.35948 / 2532-9006 / 2020.3235$

Copyright 2019 Accademia della Crusca Pubblicato con licenza creative commons CC BY-NC-ND 